\title{
Physiotherapy in the Treatment of Rheumatic Fever in Children
}

By Miss A. M. BODOANO, M.C.S.P.

In most children's hospitals and in the children's wards of General Hospitals, there are usually a number of cases of Rheumatic Fever and depending on the Physician in charge these may or may not be referred for Physiotherapy treatment.

The aims of physical treatment are to restore the child to normal activity and to prevent the disabilities and deformities likely to arise as the result of the debilitating effect of this rcondition.

In former days children were often confined to bed rest and complete inactivity up to three or six months or longer and during this time started on a slowly progressive cardiac table of exercises. Because of the cardiac involvement a too rapid or too vigorous progression could lead to increase of symptoms such as a rising temperature and heartbeat.

With the recent introduction of modern medical treatments it no longer seems to be the general practice for cases to be confined to bed for such long periods, six weeks in bed but not complete restriction of activity seems to be the more usual procedure and for this reason there is less need and therefore less demand for Physiotherapy treatment.
Even so a child whose health is below par, who has been or is suffering from a debilitating condition, who is to a variable extent restricted in activity, is likely to show a poor respiratory excursion and weak musculature and is liable to develop postural deformities.

Most cases would benefit when in hospital and if necessary afterwards by simple postural training such as, positioning in bed and at rest, breathing exercises, and some simple maintenance exercises for all muscle groups but particularly for the intrinsic muscles of the feet and the postural muscles. Many cases are kept under observation for years following the acute stage of the disease, but generally the medical practitioner is more concerned with the patient's health than with his posture and general physique, postural deformities nay be overlooked.

Every Physiotherapy Department has its quota of idiopathic scoliosis, it would be interesting to know the number of diagnosed and undiagnosed cases of Rheumatic Fever in these and other patients with postural deformities.

\section{SUGGESTED OUTLINE OF COURSE ON THERAPEUTIC MOVEMENT} 6th and 7th October, 1961

Department of Physiotherapy_University of Cape Town

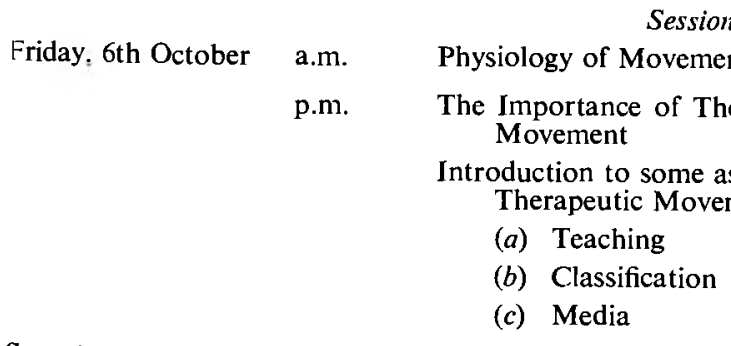

Saturday, 7th October, a.m. Demonstrations of Therapeutic Movement:

(a) Group

(b) Water

(c) Mechanical aids

p.m. Symposium on Proprioceptive Neuromuscular Facilitation Techniques

Discussion
Conducted by

Department of Physiology.

Professor Allen.

Miss M. H. S. Roper.

Staff of Department of Physiotherapy and Teaching Hospital Group.

Mr. K. Nicol.

3-4 Physiotherapists.

All lecturers and demonstrators. 